\title{
Are the preferential patterns of myocardial iron overload preserved at the CMR follow-up?
}

\author{
Antonella Meloni ${ }^{1 *}$, Vincenzo Positano ${ }^{1}$, Petra Keilberg ${ }^{1}$, Daniele De Marchi ${ }^{1}$, Gianluca Valeri ${ }^{2}$, \\ Massimiliano Missere ${ }^{3}$, Giovanni Palazzi ${ }^{4}$, Roberto Trunfio ${ }^{5}$, Massimo Lombardi ${ }^{1}$, Alessia Pepe ${ }^{1}$ \\ From 15th Annual SCMR Scientific Sessions \\ Orlando, FL, USA. 2-5 February 2012
}

\section{Background}

$\mathrm{T} 2 *$ multislice multiecho cardiac magnetic resonance (CMR) allows quantification of the segmental distribution of myocardial iron overload (MIO). This study aimed to determine if a preferential pattern of MIO was preserved between two CMR scans in thalassemia major (TM) patients.

\section{Methods}

Among the 812 TM patients with a CMR follow-up (FU) study at $18 \pm 3$ months, we selected 259 patients with significant $\mathrm{MIO}$ at baseline (global heart $\mathrm{T} 2 *<26$ $\mathrm{ms})$. Three short-axis views of the left ventricle were acquired and analyzed using a 16-segment standardized model. The T2* value on each segment was calculated, as well as the global value. Four main circumferential regions (anterior, septal, inferior and lateral) were defined.

\section{Results}

The selected patient population was divided into two groups: severe $\left(\mathrm{N}=80\right.$, global $\left.\mathrm{T} 2^{*}<10 \mathrm{~ms}\right)$ and mildmoderate MIO ( $\mathrm{N}=179$, global T2* $10-26 \mathrm{~ms})$.

For each group, there was a significant improvement in the global heart as well as in regional $\mathrm{T} 2 *$ values ( $\mathrm{P}<0.0001$ for all the pairwise comparisons).

For the whole patient population as well as for both two groups, at basal the mean $\mathrm{T} 2 *$ value over the anterior region was significantly lower than the mean $\mathrm{T} 2^{*}$ values over the other regions and the mean $\mathrm{T} 2^{*}$ over the inferior region was significantly lower than the $\mathrm{T} 2$ * values over the septal and the lateral regions. The same pattern was present at the FU, with a little difference for patients with mild-moderate MIO (see figure).

\section{Conclusions}

A preferential pattern of iron store in anterior and inferior regions was present at both basal and FU CMRs, with an increment of $\mathrm{T} 2 *$ values at FU due to a basal CMR-guided chelation therapy. The anterior region seems to be the region in which the iron accumulates first and is removed later. Our data confirm the segmental T2* cardiac MR approach useful for identifying early iron deposit and for tailoring chelation therapy.

\section{Funding}

"No-profit" support by industrial sponsorships (Chiesi, Apotex and GE Healtcare) and "Ministero della Salute, fondi ex art. 12 D.Lgs. 502/92 e s.m.i., ricerca sanitaria finalizzata anno 2006" e "Fondazione L. Giambrone".

\section{Author details}

${ }^{1}$ CMR Unit, Fondazione G.Monasterio CNR-Regione Toscana and Institute of Clinical Physiology, Pisa, Italy. ${ }^{2}$ Dipartimento di Radiologia, Azienda Ospedaliero-Universitaria Ospedali Riuniti "Umberto I-Lancisi-Salesi", Ancona, Italy. ${ }^{3}$ Departement of radiology, "John Paul II" Catholic University, Campobasso, Italy. ${ }^{4}$ Oncoematologia Pediatrica, Policlinico di Modena, Modena, Italy. ${ }^{5}$ Centro Microcitemico, U.O. di Pediatria e Neonatologia, Presidio Ospedaliero Locri - A.S.L. n. 9, Locri, Italy.

Published: 1 February 2012

doi:10.1186/1532-429X-14-S1-P190

Cite this article as: Meloni et al:: Are the preferential patterns of myocardial iron overload preserved at the CMR follow-up? Journal of Cardiovascular Magnetic Resonance 2012 14(Suppl 1):P190. 


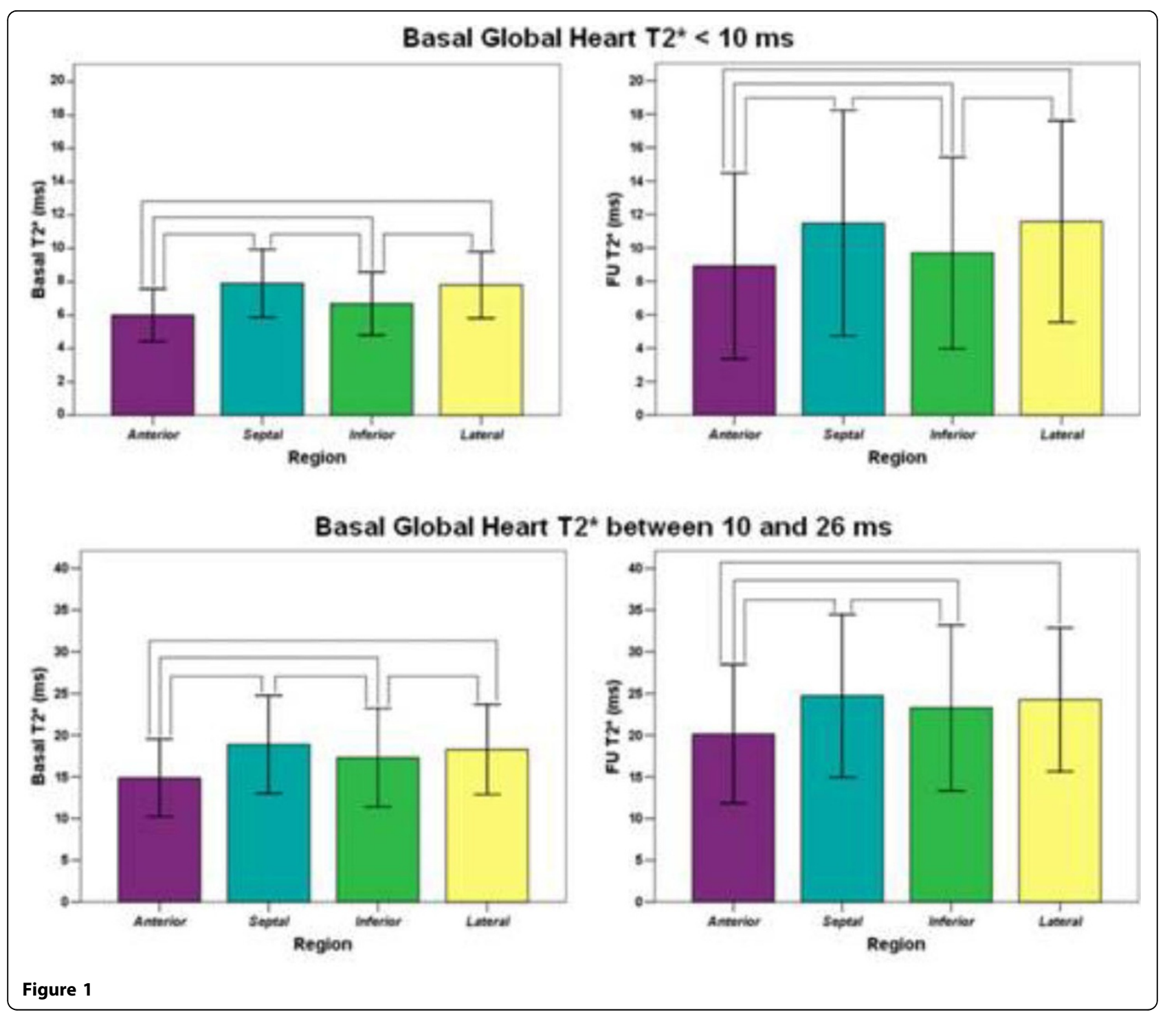

